\title{
COMPLETE CONFORMAL METRICS WITH PRESCRIBED SCALAR CURVATURE ON SUBDOMAINS OF A COMPACT MANIFOLD
}

\author{
SHIN KATO AND SHIN NAYATANI
}

\section{Dedicated to Professor Masaru Takeuchi on his sixtieth birthday}

\section{Introduction}

Let $(M, g)$ be a Riemannian manifold of dimension $n \geq 3$ and $\tilde{g}$ another metric on $M$ which is pointwise conformal to $g$. It can be written $\tilde{g}=u^{4 /(n-2)} g$, where $u$ is a positive smooth function on $M$. Then the curvature of $\tilde{g}$ is computable in terms of that of $g$ and the derivatives of $u$ up to second order. In particular, if $S$ and $\tilde{S}$ denote the scalar curvature of $g$ and $\tilde{g}$ respectively, they are related by the equation

$$
-a_{n} \Delta u+S u=\tilde{S} u^{(n+2) /(n-2)}, \quad a_{n}=\frac{4(n-1)}{n-2},
$$

where $\Delta u$ denotes the Laplacian of $u$, defined with respect to the metric $g$.

Now it is a fundamental problem to find criteria for a given function, say $f$, to be realized as the scalar curvature of some conformal metric. This is equivalent to solving the equation (1) with $f$ in place of $\tilde{S}$. Much investigation has been dedicated to this problem especially when the manifold $M$ is compact. In the noncompact case, it is geometrically natural to require the metric to be complete and then the problem amounts to seeking a solution of the above equation, which has appropriate asymptotic behavior at infinity. Also, it will be reasonable to first consider the above problem on Riemannian manifolds with simple structure at infinity. Among this class of manifolds is the complement of a closed subset in a compact Riemannian manifold. Aviles-McOwen [2] proved that, if $(M, g)$ is a compact Riemannian manifold of dimension $n$ and $\Sigma$ is a compact submanifold in $M$, the complement $\left(M \backslash \Sigma,\left.g\right|_{M \backslash \Sigma}\right)$ admits a complete conformal metric with

Received June 30, 1992. 
constant negative scalar curvature if and only if the dimension of $\sum$ is greater than $(n-2) / 2$. When $(M, g)$ is the sphere $S^{n}$ with its standard metric, this result had been known to Loewner-Nirenberg [11]. On the other hand, Schoen-Yau [21] (see also [20]) proved that if $\Sigma$ is a closed subset in $S^{n}$ and $S^{n} \backslash \Sigma$ admits a complete conformal metric with nonnegative scalar curvature, then the Hausdorff dimension of $\sum$ is less than or equal to $(n-2) / 2$.

In this paper, motivated by these results, we consider the following problems: If $(M, g)$ is a compact Riemannian manifold of dimension not less than three and $\sum$ is a closed subset in $M$, then

(a) does $M \backslash \sum$ admit complete conformal metrics with nonnegative scalar curvature?

And

(b) if it does, find criterion for a (nonnegative) function on $M \backslash \sum$ to be realized as the scalar curvature of a complete conformal metric. Can the scalar curvature be made constant?

In order to describe our results, let $\mu_{1}(M)$ denote the first eigenvalue of the operator $L=-a_{n} \Delta+S$, that is,

$$
\mu_{1}(M)=\inf \left\{\int_{M} \phi L \phi d v_{g} \mid \phi \in C^{\infty}(M), \int_{M} \phi^{2} d v_{g}=1\right\}
$$

where $d v_{g}$ is the volume element of the metric $g$. As is well-known (see [9]), the sign of $\mu_{1}(M)$ is an invariant of the conformal class of $g$, and it is positive (resp. zero, negative) if and only if the class contains a metric with everywhere positive (resp. zero, negative) scalar curvature.

In $\S 2$ we prove that if $(M, g)$ is a compact Riemannian manifold with $\mu_{1}(M) \leq 0$ and $\sum \subset M$ is a nonempty closed subset, then $M \backslash \sum$ admits no complete conformal metrics with nonnegative scalar curvature (Theorem 1). As a corollary, it follows that if $(M, g)$ is a compact Riemannian manifold with nonempty boundary, then its interior admits no such metrics.

In $\S \S 3,4$ we consider the case $\mu_{1}(M)>0$ and show that if $\Sigma \subset M$ is a compact submanifold of dimension $d \leq(n-2) / 2$, then $M \backslash \sum$ admits a variety of complete conformal metrics with nonnegative scalar curvature. In fact, letting $r$ denote the distance from a point in $M \backslash \sum$ to $\sum$, we prove that any function $f$ which decays near $\sum$ faster than $r^{2-4 d /(n-2)}$ can be realized as the scalar curvature of infinitely many complete conformal metrics on $M \backslash \sum$ (Theorem 2 (a)). In particular, $M \backslash \sum$ admits such a metric with vanishing scalar curvature. In this result, we can replace the assumption on $f$ by a weaker one, which, if $f$ does not change sign, is also necessary for $f$ to be realized as above (Theorem $2^{\prime}$ ). If $f$ is uniformly posi- 
tive or decays near $\sum$ rather mildly, it is not necessarily realized as above. We show, however, that if $d<(n-2) / 2$, there exists a complete conformal metric whose scalar curvature is positive and behaves like $r^{l}$ near $\sum$, for each $l \in[0,2$ $-4 d /(n-2)$ ) (Theorem 4). In particular, $M \backslash \sum$ admits such a metric with uniformly positive scalar curvature. In the proof of these results, we use the Green's function (on $M$ ) for $L$, being averaged over $\sum$ in one variable, to construct superand subsolutions of the equation (1) (with $f$ in place of $\tilde{S}$ ). Essential is the estimates of this function and its derivatives near $\sum$. When the closed $\Sigma$ is not a smooth manifold, our method does not work in general. However, the examples in $\S 3$ indicate that if $\sum$ supports an appropriate measure, our argument would still apply.

Acknowledgement. The second author thanks the Yukawa Foundation of Osaka University for awarding him a scholarship. Both the authors are grateful to Professor H. Ozeki for his encouragement. They also wish to thank Professor A. Kasue for his interest in this work and valuable suggestion.

During the preparation of this paper, we learned that Ma-McOwen [13] announced a result corresponding to our Corollary 2. But their proof seems different from ours.

\section{The case $\mu_{1}(M) \leq 0$}

In this section we give some nonexistence results. We first prove

Proposition 1. Let $\left(M_{0}, g_{0}\right)$ be a compact Riemannian manifold of dimension $n \geq 3$ with nompositive scalar curvature and $\Omega \subset M_{0}$ a domain such that $M_{0} \backslash \Omega \neq \emptyset$. For a complete conformal metric $g$ on $\Omega$, we set $L_{\beta}=-\Delta+\beta S$, where $\Delta$ and $S$ are the Laplacian and the scalar curvature respectively defined in terms of $g$. Then $\lambda_{1}\left(L_{\beta}\right)<0$ for $\beta \geq n / 4(n-1)(=\beta(n))$, where $\lambda_{1}\left(L_{\beta}\right)$ is defined by

$$
\lambda_{1}\left(L_{\beta}\right)=\inf \left\{\int_{\Omega} \phi L_{\beta} \phi d v_{g} \mid \phi \in C_{0}^{\infty}(\Omega), \int_{\Omega} \phi^{2} d v_{g}=1\right\} .
$$

Proof. It suffices to prove Proposition 1 for $\beta=\beta(n)$ because of the following inequality, which holds for any function $\phi$ with compact support in $\Omega$ and any $\beta \geq \beta(n)$ :

$$
\int_{\Omega}\left(|d \phi|^{2}+\beta(n) S \phi^{2}\right) \geq \frac{\beta(n)}{\beta} \int_{\Omega}\left(|d \phi|^{2}+\beta S \phi^{2}\right)
$$


If we write $g=u^{4 /(n-2)} g_{0}$, then

$$
-a_{n} \Delta\left(u^{-1}\right)+S u^{-1}=S_{0} u^{-(n+2) /(n-2)} \leq 0,
$$

where $S_{0}$ is the scalar curvature of $g_{0}$. Setting $h=u^{-n /(n-2)}$, this inequality can be rewritten as

$$
\Delta h \geq \beta(n) S h+\frac{2}{n} \frac{|d h|^{2}}{h} .
$$

Let $\phi \in C_{0}^{\infty}(\Omega)$. Then

$$
\begin{aligned}
\lambda_{1}\left(L_{\beta}\right) \int_{\Omega}(\phi h)^{2} & \leq \int_{\Omega}\left(-\phi h \Delta(\phi h)+\beta S(\phi h)^{2}\right) \\
& =\int_{\Omega}\left(-\phi h^{2} \Delta \phi-2 \phi h\langle d \phi, d h\rangle-\phi^{2} h \Delta h+\beta S(\phi h)^{2}\right) \\
& \leq \int_{\Omega}\left(-\phi h^{2} \Delta \phi-\frac{1}{2}\left\langle d\left(\phi^{2}\right), d\left(h^{2}\right)\right\rangle\right)-\frac{2}{n} \int_{\Omega} \phi^{2}|d h|^{2} .
\end{aligned}
$$

In the last inequality we have used (2). Integrating by parts we obtain

$$
\lambda_{1}\left(L_{\beta}\right) \int_{\Omega}(\phi h)^{2} \leq \int_{\Omega} h^{2}|d \phi|^{2}-\frac{2}{n} \int_{\Omega} \phi^{2}|d h|^{2} .
$$

We now let $\phi$ be a function such that $\phi=1$ on $B_{R}, \phi=0$ on $\Omega \backslash B_{2 R}$ and $|d \phi|$ $\leq 2 / R$, where $B_{R}$ is the $g$-geodesic ball of radius $R$ centered at a fixed point. Substitution in (3) gives

$$
\lambda_{1}\left(L_{\beta}\right) \int_{B_{R}} h^{2} \leq \frac{4}{R^{2}} \int_{B_{2 R}} h^{2}-\frac{2}{n} \int_{B_{R}}|d h|^{2} .
$$

Letting $R \rightarrow \infty$, the first term in the right-hand side tends to zero, since the integral is bounded above by the $g_{0}$-volume of $\Omega$. Moreover, the completeness of $g \mathrm{im}$ plies that $u$, hence $h$, is not constant. Thus $|d h|$ does not vanish identically. Therefore we can conclude

$$
\lambda_{1}\left(L_{\beta}\right) \int_{\Omega} h^{2} \leq-\frac{2}{n} \int_{\Omega}|d h|^{2}<0 .
$$

This completes the proof of Proposition 1.

Remark. Proposition 1 is a generalization to higher dimensions of a theorem of Fischer-Colbrie and Schoen ([4], Theorem 2, p. 203). In fact, our proof of Proposition 1 runs on the same lines as their proof in two dimension. 
As an immediate consequence of Proposition 1, we obtain the following

THEOREM 1. Let $(M, g)$ be a compact Riemannian manifold of dimension $n \geq 3$ with $\mu_{1}(M) \leq 0$ and $\Sigma \subset M$ a nonempty closed subset. Then $M \backslash \sum$ admits no complete conformal metrics with nonnegative scalar curvature.

Corollary 1. Let $(M, g)$ be a compact Riemannian manifold with boundary $\partial M(\neq \emptyset)$. Then $M \backslash \partial M$ admits no complete conformal metrics with nonnegative scalar curvature.

Proof. Let $(N, h)$ be a compact Riemannian manifold (without boundary) such that $M \subset N$ and $\left.h\right|_{M}=g$. By taking, if necessary, connected sum with a compact Riemannian manifold of negative scalar curvature, we may assume that $\mu_{1}(N) \leq 0$. The corollary now follows from Theorem 1 .

\section{The case $\mu_{1}(M)>0$ - scalar flat metrics}

Let $(M, g)$ be a compact Riemannian manifold of dimension $n \geq 3$ with $\mu_{1}(M)>0$. We first recall existence and basic properties of the Green's function for the conformal Laplacian:

Lemma 1. Let $(M, g)$ be as above. Then for each $y \in M$, there exists a positive integrable function $G_{y}$ on $M$ such that $L G_{y}=\delta_{y}$ in distribution sense, where $\delta_{y}$ is the Dirac measure at $y$. Such $G_{y}$ is unique and smooth in $M \backslash\{y\}$.

Moreover, there exist constants $r_{0}, c_{1}, c_{2}$ and $c_{3}$, independent of $y$, such that if $r \equiv$ $\operatorname{dist}(x, y)<r_{0}$, then

$$
\begin{gathered}
\left|G_{y}(x)-\frac{1}{a_{n}(n-2) \omega^{n-1}} r^{2-n}\right| \leq c_{1} r^{3-n}, \\
\left|d G_{y}(x)-\frac{1}{a_{n} \omega^{n-1}} r^{1-n} v_{x}^{*}\right| \leq c_{2} r^{2-n}, \\
\left|\nabla d G_{y}(x)\right| \leq c_{3} r^{-n}
\end{gathered}
$$

where $v_{x}$ denotes the unit vector at $x$ tangent to the minimal geodesic from $x$ to $y$ and $v_{x}^{*}$ its dual.

For the proof, we refer the reader to [1], Chapter 4, where only the Laplacian case is treated. But after slight modification the arguments there apply to our case. 
We now let $\Sigma \subset M$ be a compact submanifold of dimension $d$ and define a positive smooth function $G_{\Sigma}$ on $M \backslash \sum$ by

$$
G_{\Sigma}(x)=\int_{\Sigma} G_{y}(x) d \sigma_{y}, \quad x \in M \backslash \Sigma,
$$

where $d \sigma$ is the volume element of $\sum$ with respect to the induced metric. By Fubini's theorem, $G_{\Sigma}$ is integrable on $M$. It is also easy to see that $G_{\Sigma}$ is a (unique) solution of the equation $L u=\delta_{\Sigma}$, where $\delta_{\Sigma}$ is a distribution on $M$ defined by

$$
\delta_{\Sigma}(\phi)=\int_{\Sigma} \phi d \sigma, \phi \in C^{\infty}(M)
$$

Proposition 2. Suppose $d \leq n-2$. Then there exist positive constants $c_{4}, \ldots$, $c_{10}$ such that the following estimates hold near $\sum$ :

$$
\begin{gathered}
c_{4} r^{d-n+2} \leq G_{\Sigma} \leq c_{5} r^{d-n+2} \quad \text { if } d<n-2, \\
c_{6} \log \frac{1}{r} \leq G_{\Sigma} \leq c_{7} \log \frac{1}{r} \quad \text { if } d=n-2 ; \\
c_{8} r^{d-n+1} \leq\left|d G_{\Sigma}\right| \leq c_{9} r^{d-n+1}, \\
\left|\nabla d G_{\Sigma}\right| \leq c_{10} r^{d-n},
\end{gathered}
$$

where $r=\operatorname{dist}(x, \Sigma)$.

Remark. Let $\varphi$ be a positive continuous function on $\Sigma$ and define

$$
G_{\Sigma, \varphi}(x)=\int_{\Sigma} G_{y}(x) \varphi(y) d \sigma_{y}, \quad x \in M \backslash \Sigma .
$$

As will be clear from the following proof, the estimates in Proposition 2 are still valid if $G_{\Sigma}$ is replaced by $G_{\Sigma, \varphi}$.

Proof of Proposition 2. In the proof, let $c$ denote a generic constant which depends only on $M$ and $\sum$.

Let $y \in \sum$ and $(\rho, \omega)$ the geodesic polar coordinates on $\sum$ centered at $y$. Where these coordinates are defined, the volume element of $\Sigma$ is expressed as

$$
d \sigma=\theta(\rho, \omega) d \rho d \omega, \quad \theta(\rho, \omega)>0 .
$$

We take sufficiently small $\rho_{0}>0$ so that

$$
\frac{9}{10} \rho^{d-1} \leq \theta(\rho, \omega) \leq \frac{10}{9} \rho^{d-1},
$$




$$
\frac{9}{10} \rho \leq \operatorname{dist}(y, z)(\leq \rho), \quad z=(\rho, \omega)
$$

for all $y \in \sum$ and $\rho \leq \rho_{0}$.

Let $x \in M \backslash \sum$ be sufficiently near to $\Sigma$ and $y$ the point in $\sum$ nearest to $x$. Let $B_{\rho}(y)$ denote the geodesic ball in $\Sigma$ of radius $\rho$ centered at $y$. By the triangle inequality,

$$
\begin{aligned}
\operatorname{dist}(x, z) & \leq \operatorname{dist}(x, y)+\operatorname{dist}(y, z) \\
& \leq r+\rho \\
& \leq \begin{cases}2 r & \text { if } z \in B_{r}(y), \\
2 \rho & \text { if } z \in B_{\rho_{0}}(y) \backslash B_{r}(y) .\end{cases}
\end{aligned}
$$

Therefore, by (4) and (11),

$$
\begin{aligned}
G_{\Sigma}(x) & \geq \int_{B_{\rho_{0}}(y)} G_{z}(x) d \sigma_{z} \\
& \geq c \int_{B_{\rho_{0}}(y)} \operatorname{dist}(x, z)^{2-n} d \sigma_{z} \\
& \geq c \int_{B_{r^{(y)}}} r^{2-n} d \sigma_{z}+c \int_{B_{\rho_{0}(y) \backslash B_{r}(y)}} \rho^{2-n} d \sigma_{z} \\
& \geq c r^{2-n} \int_{0}^{r} \rho^{d-1} d \rho+c \int_{r}^{\rho_{0}} \rho^{d-n+1} d \rho \\
& \geq\left\{\begin{array}{l}
c r^{d-n+2} \text { if } d<n-2, \\
c \log \frac{1}{r} \text { if } d=n-2 .
\end{array}\right.
\end{aligned}
$$

The derivation of the upper estimate of $G_{\Sigma}$ is similar:

(14) $\operatorname{dist}(x, z) \geq \begin{cases}\operatorname{dist}(x, y)=r & \text { if } z \in B_{2 r}(y), \\ \operatorname{dist}(y, z)-\operatorname{dist}(x, y) \geq \frac{9}{10} \rho-r \geq \frac{2}{5} \rho & \\ & \text { if } z \in B_{\rho_{0}}(y) \backslash B_{2 r}(y),\end{cases}$

and therefore

$$
\begin{aligned}
G_{\Sigma}(x) & \leq \int_{B_{\rho_{0}}(y)} G_{z}(x) d \sigma_{z}+c \\
& \leq c \int_{B_{\rho_{0}}(y)} \operatorname{dist}(x, z)^{2-n} d \sigma_{z}+c
\end{aligned}
$$




$$
\begin{aligned}
& \leq c \int_{B_{2 \gamma}(y)} r^{2-n} d \sigma_{z}+c \int_{B_{\rho_{0}(y)} \backslash B_{2 r}(y)} \rho^{2-n} d \sigma_{z}+c \\
& \leq c r^{2-n} \int_{0}^{2 r} \rho^{d-1} d \rho+c \int_{2 r}^{\rho_{0}} \rho^{d-n+1} d \rho+c \\
& \leq \begin{cases}c r^{d-n+2} \text { if } d<n-2, \\
c \log \frac{1}{r} \text { if } d=n-2 .\end{cases}
\end{aligned}
$$

By using (5) and (6) instead of (4), the upper estimate of $\left|d G_{\Sigma}\right|$ and $\left|\nabla d G_{\Sigma}\right|$ is derived in the same way.

To derive the lower estimate of $\left|d G_{\Sigma}\right|$, we fix a constant $\alpha(>2)$ for a moment. Let $x$ and $y$ be as above and assume further that $a r<\rho_{0}$. Let $v$ be the unit vector at $x$ tangent to the minimal geodesic from $x$ to $y$. By (5), we have

$$
d G_{z}(x)(v) \geq\left\{\begin{array}{cl}
c \operatorname{dist}(x, z)^{1-n} & \text { if } z \in B_{r}(y) \\
0 & \text { if } z \in B_{\alpha r}(y) \backslash B_{r}(y), \\
-c \operatorname{dist}(x, z)^{1-n} & \text { if } z \in B_{\rho_{0}}(y) \backslash B_{\alpha r}(y)
\end{array}\right.
$$

Therefore

$$
\begin{aligned}
d G_{\Sigma}(x)(v) & \geq \int_{B_{\rho_{0}}(y)} d G_{z}(x)(v) d \sigma_{z}-c \\
& \geq c \int_{B_{\gamma}(y)} \operatorname{dist}(x, z)^{1-n} d \sigma_{z}-c \int_{B_{\rho_{0}(y)} \backslash B_{\alpha \gamma}(y)} \operatorname{dist}(x, z)^{1-n} d \sigma_{z}-c \\
& \geq c r^{1-n} \int_{0}^{r} \rho^{d-1} d \rho-c \int_{\alpha r}^{\rho_{0}} \rho^{d-n} d \rho-c(\text { by }(11),(13) \text { and }(14)) \\
& \geq c r^{d-n+1}-c(\alpha r)^{d-n+1}-c .
\end{aligned}
$$

It is clearly possible to choose $\alpha$, depending only on $M$ and $\sum$, so that the last expression is bounded below by $c \dot{r}^{d-n+1}$. This completes the proof of Proposition 2 .

COROLlary 2. Let $(M, g)$ be a compact Riemannian manifold of dimension $n \geq 3$ with $\mu_{1}(M)>0$ and $\Sigma \subset M$ a compact submanifold of dimension $d$. If $d \leq(n-2) / 2$, then $M \backslash \sum$ admits a complete conformal metric with vanishing scalar curvature.

Proof. Let $G=G_{\Sigma}$ be as above. We define a conformal metric $\tilde{g}$ on $M \backslash \sum$ by $\tilde{g}=G^{4 /(n-2)} g$. Since $L G=0$ on $M \backslash \sum, \tilde{g}$ is scalar-flat. By (7) and the assumption on $d$, we have $G \geq c r^{-(n-2) / 2}$, or $G^{4 /(n-2)} \geq c r^{-2}$, near $\sum$. Hence $\tilde{g}$ is complete. 
We recall here the formula which relates the curvature of two pointwise conformal metrics. Let $\tilde{g}=u^{4 /(n-2)} g$ and let $R$ (resp. $\tilde{R}$ ) denote the curvature tensor of $g$ (resp. $\tilde{g})$. Then

$$
\tilde{R}_{j k l}^{i}=R_{j k l}^{i}-\left(B_{k}^{i} g_{j l}-B_{j k} \delta_{l}^{i}+B_{j l} \delta_{k}^{i}-B_{l}^{i} g_{j k}\right),
$$

where

$$
B=\frac{2}{n-2} u^{-2}\left(u \nabla d u+\frac{1}{n-2}|d u|^{2} g-\frac{n}{n-2} d u \otimes d u\right)
$$

and the components are with respect to an arbitrary frame. Let $\left\{e_{i}\right\}$ be a $g$-orthonormal frame. Then $\left\{\tilde{e}_{i}=u^{-2 /(n-2)} e_{i}\right\}$ is $\tilde{g}$-orthonormal. If we let the components of $R$ (resp. $\tilde{R}$ ) be with respect to the frame $\left\{e_{i}\right\}$ (resp. $\left\{\tilde{e}_{i}\right\}$ ), then (15) is rewritten as

$$
\tilde{R}_{i j k l}=u^{-4 /(n-2)}\left\{R_{i j k l}-\left(B_{i k} \delta_{j l}-B_{j k} \delta_{i l}+B_{j l} \delta_{i k}-B_{i l} \delta_{j k}\right)\right\} .
$$

We now let $\tilde{g}$ be as in the proof of Corollary 2. Then in (16), $R_{i j k l}$ are bounded and, by Proposition 2, $\left|B_{i j}\right|$ are at most of the order $r^{-2}$ near $\sum$. Hence $\tilde{R}_{i j k l}$ are at most of the order $r^{(d-n+2)(-4 /(n-2))} r^{-2}=r^{2-4 d /(n-2)}$ near $\sum$. Thus, if $d<$ $(n-2) / 2,\left(M \backslash \sum, \tilde{g}\right)$ is an "asymptotically flat" manifold. This generalizes Schoen's construction of asymptotically Euclidean spaces (see [12], [18]).

We now summarize some results on the existence of metrics with constant scalar curvature, which are restatement or an immediate consequence of the result of Aviles-McOwen [2], our Theorem 1 and Corollary 2. Let $(M, g)$ be a compact Riemannian manifold of dimension $n \geq 3$ and $\Sigma \subset M$ a compact submanifold of dimension $d$. If $d>(n-2) / 2$, then $M \backslash \sum$ admits a complete conformal metric with constant negative scalar curvature, and if $\mu_{1}(M)>0$ and $d \leq(n-2) / 2$, then $M \backslash \sum$ admits a complete conformal metric with vanishing scalar curvature. Otherwise, that is, if $\mu_{1}(M) \leq 0$ and $d \leq(n-2) / 2, M \backslash \sum$ admits no complete conformal metrics with constant scalar curvature. This last assertion was proved by Jin [7] when $\Sigma$ is a finite set of points. In view of Theorem 4 in $\S 4$, it would be plausible to expect that if $\mu_{1}(M)>0$ and $d<(n-2) / 2$, then $M \backslash \sum$ admits a complete conformal metric with constant positive scalar curvature. This is, however, not true in general. In fact, there exist no such metrics on the sphere minus one point, as was proved by Gidas-Ni-Nirenberg [5] and Gidas-Spruck [6]. On the other hand, Schoen [19] constructed such a metric on the complement of any finite set of at least two points on the sphere. See also [14] for more existence results. 
In the following examples, we treat the case when the closed subset $\Sigma$ is not a smooth manifold but supports an appropriate measure, and show that its complement admits a complete conformal metric with vanishing scalar curvature.

Example 1. Let $(M, g)$ be the sphere $S^{n}$ with its standard metric. Fix $y \in$ $S^{n}$ and let $\alpha>0$. We choose, for $i=1,2, \ldots$, a maximal set of points $\sum_{i}=$ $\left\{y_{i}^{(j)}\right\}$ in the sphere of radius $i^{-\alpha}$ centered at $y$ such that $\operatorname{dist}\left(y_{i}^{(j)}, y_{i}^{(k)}\right) \geq i^{-\alpha-1}$ if $j \neq k$. The number of points in $\sum_{i}$ is then of the order $i^{n-1}$. Let $\Sigma=U \Sigma_{i} U$ $\{y\}$. Schoen-Yau [21] observed that if $\alpha<1$ there exist no complete conformal metrics on $S^{n} / \sum$ with nonnegative scalar curvature and with bounded curvature.

We now show that if $\alpha>(n+2) /(n-2), S^{n} \backslash \sum$ admits a complete conformal metric with vanishing scalar curvature. Let $a_{i}=i^{-n-\varepsilon}$ for $\varepsilon>0$ and define

$$
G_{\Sigma}(x)=\sum_{i=1}^{\infty} a_{i}\left(\sum_{j} G_{y_{i}(j)}(x)\right), x \in S^{n} \backslash \sum .
$$

Since $\sum_{i=1}^{\infty} a_{i} i^{n-1}<\infty$, the right-hand side converges up to derivatives. Hence $L G_{\Sigma}=0$ and so $\tilde{g}=\left(G_{\Sigma}\right)^{4 /(n-2)} g$ is a scalar-flat metric on $S^{n} \backslash \sum$. Let $r=r(x)$ $=\operatorname{dist}(x, \Sigma)$. For any $x \in S^{n} \backslash \sum$ there exists $y_{i}^{(j)}$ such that $r=\operatorname{dist}\left(x, y_{i}{ }^{(j)}\right)$. Then we have $r \leq c i^{-\alpha-1}$, where $c$ is a constant independent of $x$. Therefore $a_{i} \geq$ $c r^{(n+\varepsilon) /(\alpha+1)}$ and

$$
\begin{aligned}
G_{\Sigma}(x) & \geq a_{i} G_{y_{i}(j)}(x) \\
& \geq c a_{i} r^{2-n} \\
& \geq c r^{(n+\varepsilon) /(\alpha+1)+2-n} .
\end{aligned}
$$

If $\alpha>(n+2) /(n-2)$, then choosing $\varepsilon$ sufficiently small we have $(n+\varepsilon) /(\alpha+1)$ $+2-n \leq-(n-2) / 2$ so that $G_{\Sigma} \geq c r^{-(n-2) / 2}$ near $\sum$. Hence $\tilde{g}$ is complete.

Example 2. Let $\Gamma$ be a Kleinian group, that is, a discrete subgroup of the group of conformal diffeomorphisms of $S^{n}$. Let $\Lambda=\Lambda(\Gamma)$ denote the limit set of $\Gamma$ and $\delta=\delta(\Gamma)$ the critical exponent of $\Gamma$. For the terminologies above and in the following, we refer the reader to [17]. We only note here that $\delta$ coincides with the Hausdorff dimension of $\Lambda$ for a certain class of $\Gamma$. Let $\mu$ be a Patterson-Sullivan density, which is a measure on $S^{n}$ with support on $\Lambda$, and define

$$
G_{\Lambda}(x)=\int_{\Lambda} G_{y}(x) d \mu_{y}, \quad x \in S^{n} \backslash \Lambda
$$

Then $L G_{\Lambda}=0$ and $\tilde{g}=\left(G_{\Lambda}\right)^{4 /(n-2)} g$ is a scalar-flat metric on $S^{n} \backslash \Lambda$. We now assume that $\Gamma$ is convex co-compact. Then the measure $\mu$ coincides, up to a con- 
stant multiple, with the $\delta$-dimensional Hausdorff measure restricted to $\Lambda$ and there exist constants $c, C, \rho_{0}$ such that if $y \in \Lambda$ and $\rho \leq \rho_{0}$ then

$$
c \leq \mu\left(B_{\rho}(y)\right) / \rho^{\delta} \leq C,
$$

where $B_{\rho}(y)$ is the ball in $S^{n}$ of radius $\rho$ centered at $y$ (see [17], pp. 82-84). Let $x \in S^{n} \backslash \Lambda$ be sufficiently near to $\Lambda, r=\operatorname{dist}(x, \Lambda)$ and $y$ the point in $\Lambda$ nearest to $x$. By (7) and (17), we have

$$
\begin{aligned}
G_{\Lambda}(x) & \geq c \int_{B_{r}(y)} \operatorname{dist}(x, z)^{2-n} d \mu_{z} \\
& \geq c r^{2-n} \mu\left(B_{r}(y)\right) \\
& \geq c r^{\delta-n+2} .
\end{aligned}
$$

Hence, if $\delta \geq(n-2) / 2, \tilde{g}$ is complete.

\section{The case $\mu_{1}(M)>0$ - prescribing scalar curvature}

Let $(M, g)$ be as in $\S 3$ and $\Sigma \subset M$ a compact submanifold of dimension $d$. In Theorem 2 below we prove that, if $d \leq(n-2) / 2$, any function on $M \backslash \Sigma$ which decays rapidly enough near $\sum$ can be realized as the scalar curvature of a complete conformal metric on $M \backslash \sum$. To do this we need the following

Lemma 2. Let $(M, g)$ be a Riemannian manifold and $F(x, u)$ a smooth function on $M \times \mathbf{R}_{+}$. Suppose there exist functions $u_{+}$and $u_{-}$such that $u_{+} \geq u_{-}>0$ and

$$
L u_{+} \geq F\left(x, u_{+}\right), \quad L u_{-} \leq F\left(x, u_{-}\right) \text {on } M .
$$

Then the equation

$$
L u=F(x, u)
$$

admits a solution $u$ satisfying $u_{-} \leq u \leq u_{+}$.

The function $u_{+}$(resp. $u_{-}$) is referred to as a supersolution (resp. subsolution) of the equation (18). This lemma is now standard among the experts and we omit the proof (see [9], [16], for example).

THEOREM 2. Let $(M, g)$ be a compact Riemannian manifold of dimension $n \geq 3$ with $\mu_{1}(M)>0, \Sigma \subset M$ a compact submanifold of dimension $d \leq(n-2) / 2$ and $r$ 
the distance from a point in $M \backslash \sum$ to $\sum$.

(a) Let $f$ be a smooth function on $M \backslash \sum$ which satisfies

$$
|f| \leq c r^{l} \text { near } \Sigma
$$

for some constants $c>0$ and $l>2-4 d /(n-2)$. Then there exist infinitely many complete conformal metrics on $M \backslash \sum$ whose scalar curvature is equal to $f$ and whose ratio to $g$ is of the order $r^{-(4-4 d /(n-2))}$ near $\sum$.

(b) Let $f$ be a nonpositive smooth function on $M \backslash \sum$ which satisfies

$$
-c^{\prime} r^{l^{\prime}} \leq f \leq-c r^{l} \text { near } \sum
$$

for some constants $c>0, c^{\prime}>0$ and $l \geq l^{\prime}>2-4 d /(n-2)$. Then there exists at least one complete conformal metric on $M \backslash \sum$ whose scalar curvature is equal to $f$ and whose ratio to $g$ is at least of the order $r^{-\left(l^{\prime}+2\right)}$ near $\sum$.

Proof. We may assume without loss of generality that the scalar curvature $S$ of the metric $g$ is positive everywhere on $M$. Recall that the metric $u^{4 /(n-2)} g$ has scalar curvature $f$ if and only if $u$ satisfies the equation

$$
L u=f u^{(n+2) /(n-2)} .
$$

Let $G=G_{\Sigma}$ be as above. To prove (a) we define functions $u_{ \pm}$on $M \backslash \sum$ by

$$
u_{+}=\gamma G\left(1+G^{-\alpha}\right), u_{-}=\gamma G\left(1-\beta G^{-\alpha}\right),
$$

where $0<\alpha<1, \gamma>0$ and $\beta=(\min G)^{\alpha} / 2$. The constants $\alpha$ and $\gamma$ will be determined later. By direct computation, we have

$$
\begin{aligned}
u_{+}^{-(n+2) /(n-2)} L u_{+} & =\alpha \gamma^{-4 /(n-2)} G^{-2 n /(n-2)-\alpha}\left(1+G^{-\alpha}\right)^{-(n+2) /(n-2)} \\
& \times\left(S G^{2}+a_{n}(1-\alpha)|d G|^{2}\right) .
\end{aligned}
$$

The right-hand side is positive on $M \backslash \sum$ and, by Proposition 2, is estimated from below by

$$
\begin{gathered}
c(\alpha) \gamma^{-4 /(n-2)} r^{(d-n+2)(-2 n /(n-2)-\alpha)} r^{2(d-n+1)} \\
=c(\alpha) \gamma^{-4 /(n-2)} r^{2-4 d /(n-2)+\alpha(n-2-d)}
\end{gathered}
$$

near $\sum$, where $c(\alpha)$ is a constant which depends only on $\alpha$. It is now possible to choose $\alpha>0$ so that $l \geq 2-4 d /(n-2)+\alpha(n-2-d)$ and hence

$$
u_{+}^{-(n+2) /(n-2)} L u_{+} \geq c(\alpha) \gamma^{-4 /(n-2)} r^{l}
$$

near $\sum$. By taking $\gamma$ sufficiently small, we can finally realize $u_{+}^{-(n+2) /(n-2)} L u_{+} \geq f$ 
everywhere on $M \backslash \sum$. Thus $u_{+}$is a supersolution of the equation (19). We can similarly show that, by choosing $\alpha$ and then $\gamma$ sufficiently small, $u_{-}$gives a subsolution of (19). It is clearly possible to choose $\alpha$ and $\gamma$ so that $u_{+}$and $u_{-}$simultaneously give super- and subsolutions respectively. Since $u_{-} \leq u_{+}$, we can find, by Lemma 2, a solution $u$ of (19) such that $u_{-} \leq u \leq u_{+}$. Thus the metric $u^{4 /(n-2)} g$ has scalar curvature $f$. Moreover, $u_{+}$and $u_{-}$are of the order $r^{-(n-2-d)}$ near $\sum$ and so is $u$. This implies the completeness of $u^{4 /(n-2)} g$. Since $r$ can be arbitrarily small, there exist infinitely many such metrics. This finishes the proof of (a).

To prove (b) we define functions $u_{ \pm}$on $M \backslash \sum$ by

$$
u_{+}=\gamma G^{p}\left(1+\beta G^{-\alpha}\right), u_{-}=\gamma^{\prime} G^{p^{\prime}},
$$

where $p=(n-2)(l+2) / 4(n-2-d), p^{\prime}=(n-2)\left(l^{\prime}+2\right) / 4(n-2-d)$ and $\alpha$ is a fixed constant such that $p-1<\alpha<p$. Notice that $p \geq p^{\prime}>1$ and so $\alpha>0$. The constants $\beta, \gamma$ and $\gamma^{\prime}$ are positive and to be determined later. By direct computation, we have

$$
\begin{gathered}
u_{+}^{-(n+2) /(n-2)} L u_{+}=\gamma^{-4 /(n-2)} G^{-4 p /(n-2)-2}\left(1+\beta G^{-\alpha}\right)^{-(n+2) /(n-2)} \\
\times\left\{-(p-1) S G^{2}-a_{n} p(p-1)|d G|^{2}+\beta(\alpha-p+1) S G^{2-\alpha}\right. \\
\left.+a_{n} \beta(p-\alpha)(\alpha-p+1) G^{-\alpha}|d G|^{2}\right\} .
\end{gathered}
$$

By taking $\beta$ sufficiently large, we can realize $u_{+}^{-(n+2) /(n-2)} L u_{+} \geq 0(\geq f)$ away from $\sum$. On the other hand, by Proposition 2, the right-hand side is estimated from below by

$$
\begin{gathered}
-c r^{-4 /(n-2)} r^{(d-n+2)(-4 p /(n-2)-2)} r^{2(d-n+1)} \\
=-c r^{-4 /(n-2)} r^{l}
\end{gathered}
$$

near $\sum$. We now take $\gamma$ sufficiently large so that $u_{+}^{-(n+2) /(n-2)} L u_{+} \geq f$ near $\sum$. Thus $u_{+}$is a supersolution of the equation (19). It is an easy matter to verify that if $\gamma^{\prime}$ is chosen sufficiently small, $u_{-}$gives a subsolution of (19) and satisfies $u_{-}$ $\leq u_{+}$. By repeating the same argument as in the proof of (a), we get a metric with the required properties. The proof of Theorem 2 is now complete.

Remark. Let $\tilde{g}=\left(G_{\Sigma}\right)^{4 /(n-2)} g$, the metric on $M \backslash \sum$ as in the proof of Corollary 2 , and $\tilde{r}$ the distance to a fixed point in $M \backslash \sum$ with respect to $\tilde{g}$. Then it can be shown, by a standard geometric argument, that $\tilde{r}$ is of the order $r^{-(1-2 d /(n-2))}$ near $\sum$ if $d<(n-2) / 2$ and of the order $-\log r$ if $d=(n-2) / 2$. Thus, if $d<(n-2) / 2$, the condition on $f$ in Theorem 2 (a) can be expressed in terms of $\tilde{r}$ as " $|f| \leq c \tilde{r}^{-s}$ near infinity for some $s>2$ ". Now let $\hat{g}$ denote the metric 
$u^{4 /(n-2)} g$ in the proof of Theorem 2 (a) and write $\hat{g}=v^{4 /(n-2)} \tilde{g}$. Then $v\left(=u / G_{\Sigma}\right)$ is bounded below and above by positive constants. Moreover, we have an estimate

$$
|v-r| \leq c r^{\alpha(n-2-d)}
$$

near $\sum$, where $\alpha$ and $\gamma$ are the constants in the proof. In view of this estimate, it is interesting to observe that we can choose

$$
\alpha=\left\{\begin{array}{cl}
\left(l-\left(2-\frac{4 d}{n-2}\right)\right) /(n-2-d) & \text { if } l<n-\frac{n+2}{n-2} d, \\
1-\varepsilon & \text { if } n-\frac{n+2}{n-2} d \leq l<n-\frac{n+2}{n-2} d+2, \\
1 & \text { if } n-\frac{n+2}{n-2} d+2 \leq l,
\end{array}\right.
$$

where $\varepsilon$ may be arbitrarily small.

If $(M, g)$ is the sphere and $\Sigma$ is a point, $\left(M \backslash \sum, \tilde{g}\right)$ is isometric to the Euclidean space. In this particular case, the above discussion shows that Theorem 2 (a) is reduced to a result of $\mathrm{Ni}$ ([16], Theorem 1.4, p. 494, see also [10], [15]).

The proof of Theorem 2 (b) also implies the following result by simply choosing $l^{\prime}=0$ :

THEOREM 3. Let $(M, g)$ be a compact Riemannian manifold of dimension $n \geq 3$ with $\mu_{1}(M)>0, \Sigma \subset M$ a compact submanifold of dimension $d,(n-2) / 2<d$ $<n-2$, and $r$ the distance from a point in $M \backslash \sum$ to $\sum$. Let $f$ be a bounded nonpositive smooth function on $M \backslash \sum$ which satisfies

$$
f \leq-c r^{l} \text { near } \Sigma
$$

for some constants $c>0$ and $l \geq 0$. Then there exists a complete conformal metric on $M \backslash \sum$ whose scalar curvature is equal to $f$.

The assumption on $f$ in Theorem 2 (a) can be weakened. In fact, $f$ need not decay uniformly near $\sum$ as Theorem 2 ' below indicates (see [8] for related results). The proof of the second assertion borrows a technique from [3].

THEOREM 2'. Let $(M, g), \sum$ and $r$ be as in Theorem 2. Let $f$ be a smooth function on $M \backslash \sum$ which satisfies the following condition for some constant $c$ : 


$$
\int_{M \backslash \Sigma} G_{x}(y)|f(y)| r(y)^{(d-n+2)(n+2) /(n-2)} d v_{y} \leq c r(x)^{d-n+2}, \quad x \in M \backslash \sum .
$$

Then there exist infinitely many complete conformal metrics on $M \backslash \sum$ whose scalar curvature is equal to $f$ and whose ratio to $g$ is of the order $r^{-(4-4 d /(n-2))}$ near $\sum$. Moreover, if $f$ does not change sign, the condition (20) is necessary for the existence of such a metric.

Proof. Let

$$
\Phi(x)=\frac{1}{G_{\Sigma}(x)} \int_{M \backslash \Sigma} G_{x}(y)|f(y)| G_{\Sigma}(y)^{(n+2) /(n-2)} d v_{y}, \quad x \in M \backslash \Sigma .
$$

By (7), the condition (20) is equivalent to the boundedness of $\Phi$.

We now prove the first assertion. Since $|f|$ is locally Lipschitz, $\Phi$ is twice continuously differentiable and $L(G \Phi)=|f| G^{(n+2) /(n-2)}$, where $G=G_{\Sigma}$. We define functions $u_{ \pm}$on $M \backslash \sum$ by

$$
u_{+}=\gamma G(1+\Phi), \quad u_{-}=\gamma G(1-\beta \Phi),
$$

where $\beta=(2 \sup \Phi)^{-1}$ and $\gamma>0$ will be determined later. We compute

$$
\begin{aligned}
u_{+}^{-(n+2) /(n-2)} L u_{+} & =\gamma^{-4 /(n-2)}(1+\Phi)^{-(n+2) /(n-2)}|f| \\
& \geq \gamma^{-4 /(n-2)}(1+\sup \Phi)^{-(n+2) /(n-2)}|f| .
\end{aligned}
$$

By choosing $\gamma$ so that $\gamma \leq(1+\sup \Phi)^{-(n+2) / 4}$, we obtain $u_{+}^{-(n+2) /(n-2)} L u_{+} \geq f$. Similarly,

$$
\begin{aligned}
u_{-}^{-(n+2) /(n-2)} L u_{-} & =-\gamma^{-4 /(n-2)}(1-\beta \Phi)^{-(n+2) /(n-2)} \beta|f| \\
& \leq-\gamma^{-4 /(n-2)} \beta|f| . \\
& \leq f
\end{aligned}
$$

if $\gamma \leq \beta^{(n-2) / 4}=(2 \sup \Phi)^{-(n-2) / 4}$. The argument as in the proof of Theorem 2 (a) finishes the proof of the first assertion.

To prove the second assertion, let $\left\{\Omega_{j}\right\}_{j=1}^{\infty}$ be a sequence of relatively compact domains in $M \backslash \sum$ with smooth boundary such that $\Omega_{1} \subset \subset \Omega_{2} \subset \subset \cdots$ and $\cup_{j=1}^{\infty} \Omega_{j}=M \backslash \sum$. For $x \in \Omega_{j}$, let $G_{x}^{(j)}$ be the Dirichlet Green's function on $\Omega_{j}$ for $L$ with pole at $x$. We define

$$
\Phi^{(j)}(x)=\frac{1}{G_{\Sigma}(x)} \int_{\Omega_{j}} G_{x}^{(j)}(y)|f(y)| G_{\Sigma}(y)^{(n+2) /(n-2)} d v_{y}, \quad x \in \Omega_{j} .
$$


Then $G_{\Sigma} \Phi^{(j)}$ is a unique solution of the equation

$$
L w=|f| G^{(n+2) /(n-2)} \text { in } \Omega_{j}, w=0 \text { on } \partial \Omega_{j} .
$$

where $G=G_{\Sigma}$. As $j$ tends to infinity, $G_{x}^{(j)}$ converges to the minimal Green's function on $M \backslash \sum$. But this coincides with $G_{x}$, the Green's function on $M$, since $\Sigma$ has codimension not less than two (see [21], p. 55). Lebesgue's convergence theorem then implies that $\Phi^{(j)}(x)$ converges to $\Phi(x)$.

Now suppose that the equation $L u=f u^{(n+2) /(n-2)}$ admits a positive solution $u$ which is of the order $r^{d-n+2}$ near $\sum$. By (7), $v=u / G$ is bounded below and above by positive constants; $0<c_{1} \leq v \leq c_{2}$. We first consider the case $f \geq 0$. We have

$$
L(G v)=f(G v)^{(n+2) /(n-2)} \geq c_{1}^{(n+2) /(n-2)} f G^{(n+2) /(n-2)},
$$

and thus

$$
L\left(c_{1}^{-(n+2) /(n-2)} G v\right) \geq f G^{(n+2) /(n-2)}
$$

on $M \backslash \sum$. On the other hand,

$$
L\left(c_{1}^{-4 /(n-2)} G\right)=0 \leq f G^{(n+2) /(n-2)} .
$$

Since $c_{1}^{-4 /(n-2)} G \leq c_{1}^{-(n+2) /(n-2)} G v$, it follows from Lemma 2 that there exists a solution $w_{1}$ of the equation $L w=f G^{(n+2) /(n-2)}$ such that $c_{1}^{-4 /(n-2)} G \leq w_{1} \leq$ $c_{1}^{-(n+2) /(n-2)} G v\left(\leq c_{1}^{-(n+2) /(n-2)} c_{2} G\right)$. Therefore

$$
L\left(w_{1}-G \Phi^{(j)}\right)=0 \text { in } \Omega_{j}, \quad w_{1}-G \Phi^{(j)}=w_{1} \geq 0 \text { on } \partial \Omega_{j},
$$

and the maximum principle implies

$$
\Phi^{(j)} \leq w_{1} / G \leq c_{1}^{-(n+2) /(n-2)} c_{2}
$$

in $\Omega_{j}$. Hence $\Phi \leq c_{1}^{-(n+2) /(n-2)} c_{2}$ on $M \backslash \sum$.

We now treat the case $f \leq 0$. The same argument as above shows the existence of a solution $w_{2}$ of the equation $L w=f G^{(n+2) /(n-2)}$ such that $c_{1}^{-(n+2) /(n-2)} G v$ $\leq w_{2} \leq c_{1}^{-(n+2) /(n-2)} c_{2} G$. Letting $w_{3}=c_{1}^{-(n+2) /(n-2)} c_{2} G-w_{2}$, we have $L w_{3}=$ $-f G^{(n+2) /(n-2)}=|f| G^{(n+2) /(n-2)}$ and $0 \leq w_{3} \leq c_{1}^{-(n+2) /(n-2)} G\left(c_{2}-v\right)\left(\leq c_{1}^{-(n+2) /(n-2)}\right.$. $\left.\left(c_{2}-c_{1}\right) G\right)$, and hence

$$
L\left(w_{3}-G \Phi^{(j)}\right)=0 \text { in } \Omega_{j}, \quad w_{3}-G \Phi^{(j)}=w_{3} \geq 0 \text { on } \partial \Omega_{j} .
$$

The boundedness of $\Phi$ now follows in the same way as above. This completes the proof of Theorem $2^{\prime}$. 
Remark. The second assertion in Theorem $2^{\prime}$ fails to hold if we drop the assumption that $f$ does not change sign. To see this, we first observe that on $\mathbf{R}^{n}$, which is conformally diffeomorphic to the sphere minus one point, the function $\Phi$ in the above proof coincides (up to a constant multiple) with

$$
\Psi(x)=\int_{\mathbf{R}^{n}}|x-y|^{2-n}|f(y)| d y .
$$

Also, the existence of a metric with the required properties is equivalent to the existence of a solution of the equation $-a_{n} \Delta v=f v^{(n+2) /(n-2)}$ on $\mathbf{R}^{n}$ which is bounded below and above by positive constants. We now let $v=2+\sin x_{1}$. Then

$$
\begin{aligned}
f & =-a_{n} v^{-(n+2) /(n-2)} \Delta v \\
& =a_{n}\left(2+\sin x_{1}\right)^{-(n+2) /(n-2)} \sin x_{1},
\end{aligned}
$$

which clearly changes sign. Since $|f| \geq c\left|\sin x_{1}\right| \geq c(\varepsilon)>0$ on $\left\{\varepsilon \leq x_{1} \leq \pi-\varepsilon\right\}$, we obtain

$$
\begin{aligned}
\Psi(x) & =\int_{\mathbf{R}^{n}}|y|^{2-n}|f(x-y)| d y \\
& \geq c \int_{\left\{\varepsilon-x_{1} \leq y_{1} \leq \pi-\varepsilon-x_{1}\right\}}|y|^{2-n} d y \\
& =\infty .
\end{aligned}
$$

In Theorem 2 (a) the assumption on the power $l$ is essential; if $l \leq 2-$ $4 d /(n-2), f$ is not necessarily the scalar curvature of a complete conformal metric whose ratio to $g$ is of the order $r^{-(4-4 d /(n-2))}$ near $\sum$. Without this last restriction on the metric we seek, however, we do not know whether the assumption is optimal or not. On the other hand, we have the following

TheOREM 4. Let $(M, g)$ be a compact Riemannian manifold of dimension $n \geq 3$ with $\mu_{1}(M)>0$. Let $\Sigma \subset M$ be a compact submanifold of dimension $d<(n-2) / 2$ and $r$ the distance from a point in $M \backslash \sum$ to $\sum$. For any $l \in[0,2-4 d /(n-2))$, there exists a complete conformal metric on $M \backslash \sum$ whose scalar curvature is positive and of the order $r^{l}$ near $\sum$. In particular, $M \backslash \sum$ admits a complete conformal metric whose scalar curvature is bounded below and above by positive constants.

Proof. Again we assume that $S$, the scalar curvature of $g$, is positive. Let $u=G^{p}$ where $G=G_{\Sigma}$ and $p=(n-2)(l+2) / 4(n-2-d)(<1)$. Let $\tilde{g}=$ $u^{4 /(n-2)} g$. Since, by $(7), u$ is at least of the order $r^{-(n-2) / 2}$ near $\sum, \tilde{g}$ is complete. 
Moreover, its scalar curvature is given by

$$
u^{-(n+2) /(n-2)} L u=(1-p) G^{-4 p /(n-2)-2}\left(S G^{2}+a_{n} p|d G|^{2}\right) .
$$

The right-hand side is positive and, by Proposition 2, of the order $r^{l}$ near $\sum$. This completes the proof of Theorem 4.

Addendum. During the submission of this paper, Professor R. McOwen informed us that Theorem 1 and Theorem 2 (a) were obtained independently by Delanoë [22]. But our proof of Theorem 1 is different from his.

\section{REFERENCES}

[1] T. Aubin, Nonlinear analysis on manifolds. Monge-Ampère equations, Springer, New York, 1982.

[2] P. Aviles and R. C. McOwen, Complete conformal metrics with negative scalar curvature in compact Riemannian manifolds, Duke Math. J., 56 (1988), 395-398.

[ 3 ] H. Brezis and S. Kamin, Sublinear elliptic equations in $\mathbf{R}^{n}$, Manuscripta Math., $\mathbf{7 4}$ (1992), 87-106.

[4] D. Fischer-Colbrie and R. Schoen, The structure of complete stable minimal surfaces in 3-manifolds of non-negative scalar curvature, Comm. Pure Appl. Math., 33 (1980), 199-211.

[5] B. Gidas, W.-M. Ni and L. Nirenberg, Symmetry and related properties via the maximum principle, Comm. Math. Phys., 68 (1979), 209-243.

[6] B. Gidas and J. Spruck, Global and local behavior of positive solutions of non-linear elliptic equations, Comm. Pure Appl. Math., 34 (1981), 525-598.

[7] Z. Jin, A counterexample to the Yamabe problem for complete noncompact manifolds, Partial Differential Equations (Lecture Notes in Math. 1306) ed. by S. S. Chern, Springer, Berlin, 1988, 93-101.

[8] S. Kato, Conformal deformation to prescribed scalar curvature on complete noncompact Riemannian manifolds with nonpositive curvature, Tôhoku Math. J., 45 (1993), 205-230.

[9] J. Kazdan and F. Warner, Scalar curvature and conformal deformation of Riemannian structure, J. Differential Geom., 10 (1975), 113-134.

[10] Y. Li and W.-M. Ni, On conformal scalar curvature equations in $\mathbf{R}^{n}$, Duke Math. J., 57 (1988), 895-924.

[11] C. Loewner and L. Nirenberg, Partial differential equations invariant under conformal and projective transformations, Contributions to Analysis, Academic Press, New York, 1974, 245-272.

[12] J. M. Lee and T. H. Parker, The Yamabe problem, Bull. Amer. Math. Soc., 17 (1987), 37-91.

[13] X. Ma and R. C. McOwen, The Laplacian on complete manifolds with warped cylindrical ends, Comm. Partial Differential Equations, 16 (1991), 1583-1614.

[14] R. Mazzeo and N. Smale, Conformally flat metrics of constant positive scalar curvature on subdomains of the sphere, J. Differential Geom., 34 (1991), 581-621.

[15] M. Naito, A note on bounded positive entire solutions of semilinear equations, 
Hiroshima Math. J., 14 (1984), 211-214.

[16] W. M. Ni, On the elliptic equation $\Delta u+K(x) u^{(n+2) /(n-2)}=0$, its generalizations, and applications in geometry, Indiana Univ. Math. J., 31 (1982), 493-529.

[17] P. J. Nicholls, The ergodic theory of discrete groups, Cambridge University Press, Cambridge, 1989.

[18] R. Schoen, Conformal deformation of a Riemannian metric to constant scalar curvature, J. Differential Geom., 20 (1984), 479-495.

[19] R. Schoen, The existence of weak solutions with prescribed singular behavior for a conformally invariant scalar equation, Comm. Pure Appl. Math., 41 (1988), $317-392$.

[20] R. Schoen, A report on some recent progress on nonlinear problems in geometry, Surveys in Differential Geom., 1 (1991), 201-241.

[21] R. Schoen and S.-T. Yau, Conformally flat manifolds, Kleinian groups and scalar curvature, Invent. Math., 92 (1988), 47-71.

[22] P. Delanoë, Generalized stereographic projections with prescribed scalar curvature, Contemporary Math., 127 (1992), 17-25.

S. Kato

Department of Mathematics

Nara Women's University

Nara 630

Japan

S. Nayatani

Mathematical Institute

Tôhoku University

Sendai 980

Japan 